\title{
'Tantalizing' hints of room-temperature superconductivity
}

\section{Doped graphite may superconduct at more than $100^{\circ} \mathrm{C}$.}

\section{Edwin Cartlidge}

18 September 2012

Researchers in Germany have claimed a breakthrough: a material that can act as a superconductor - transmit electricity with zero resistance - at room temperature and above. Superconductors offer huge potential energy savings, but until now have worked only at temperatures of lower than about $-110^{\circ} \mathrm{C}$.

Now, Pablo Esquinazi and his colleagues at the University of Leipzig report that flakes of humble graphite soaked in water seem to continue superconducting at temperatures of greater than $100{ }^{\circ} \mathrm{C}^{1}$. Even Esquinazi admits that the claim "sounds like science fiction", but the work has been published in the peer-reviewed journal Advanced Materials, and other physicists contacted by Nature say that the results, although tentative, merit further scrutiny.

Graphite, which consists of layers of carbon atoms arranged in hexagonal lattices,

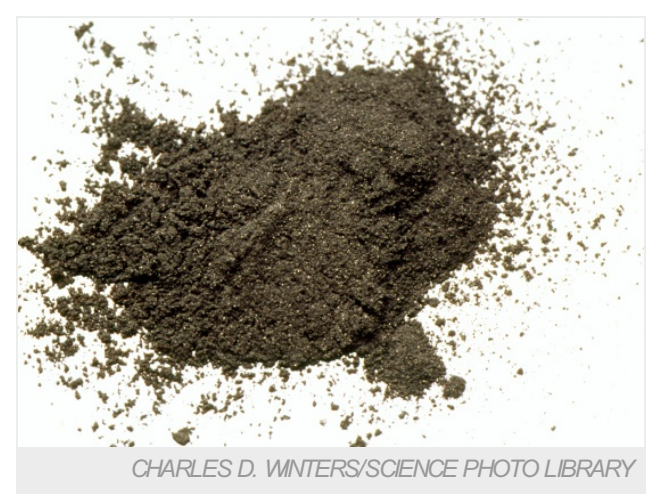

Simple graphite, if doped with distilled water, may be able to act as a superconductor at room temperature. can superconduct when doped with elements that provide it with additional free electrons. Calcium graphite, for example, superconducts at up to 11.5 kelvin (about $-260{ }^{\circ} \mathrm{C}$ ) ${ }^{2}$, and theorists have predicted that temperatures of up to 60 kelvin could be reached if enough free electrons were available.

Esquinazi's team speculates that high concentrations of electrons form at the interfaces between neighbouring thin segments of graphite. Having already observed superconductivity at more than 100 kelvin at the interfaces within an artificial type of bulk graphite known as pyrolytic graphite ${ }^{3}$, the researchers wondered whether they could reach even higher temperatures by doping flakes of graphite powder.

The first dopant they tried was ordinary water. They got lucky. They placed 100 milligrams of pure graphite powder made up of flakes a few hundredths of a millimetre long and tens of nanometres thick into 20 millilitres of distilled water. After stirring the mixture for around 23 hours, they filtered out the powder and then dried it overnight at $100{ }^{\circ} \mathrm{C}$. They found that after being placed in a magnetic field, each sample would remain slightly magnetized after the field was removed.

Esquinazi says that this tiny residual magnetization is a sign of either superconductivity or ordinary ferromagnetism. To find out whether they were seeing the former, his team investigated how the magnetization varied with the strength of the applied field and with temperature. The resultant plots were very similar to those for the first high-temperature oxide superconductors, discovered in the 1980s.

\section{High hopes}

Esquinazi concedes that his evidence is "tantalizing" rather than watertight. For one thing, his group has not been able to show that its samples actually conduct electricity with zero resistance. The researchers tried to do this by compressing the soaked powder into pellets to force the grains into electrical contact, but found that this caused the superconducting effect to disappear. Also, they have not been able to prove that the magnetic fields are absent from the interior of the flakes - a fundamental characteristic of superconductors.

In addition, the samples do not lose their apparent superconductivity as they heat up. The team reports that the superconducting state remained at temperatures up to about 400 kelvin, or around $130{ }^{\circ} \mathrm{C}$, and that a simple extrapolation of the data indicates an upper limit of around 1,000 kelvin. Esquinazi says that since writing the paper, the team has in fact observed signs of superconductivity up to 500 kelvin, but that at this point the heat starts to degrade the samples, altering their magnetization and making it difficult to observe the transition to a non-superconducting state. 
magnetic data at low temperatures "look very much like the signal you'd get from a superconductor" but that he is puzzled by the change in behaviour at higher temperatures. He says he would have expected the residual magnetization to be greatly reduced at 300 kelvin unless "the transition temperature really is way above that".

Meanwhile, Alexander Gurevich, a theorist at Old Dominion University in Norfolk, Virginia, cautions that previous claims of hightemperature superconductivity have come unstuck when scrutinized. The magnetic response could have been generated by impurities that were introduced during the sample treatment, he says. But he adds that if confirmed, the finding could have "far-reaching consequences".

Nature | doi:10.1038/nature.2012.11443

\section{References}

1. Scheike, T. et al. Adv. Mater. advance online publication http://dx.doi.org/10.1002/adma.201202219 (2012).

2. Weller, T. et al. Nature Physics, 1, 39-41 (2005).

3. Ballestar, A., Barzola-Quiquia, J. \& Esquinazi, P. preprint at http://arxiv.org/abs/1206.2463 (2012). 